\title{
NECESSARY CONDITIONS FOR CONSTRAINED DISTRIBUTED PARAMETER SYSTEMS WITH DEVIATING ARGUMENT
}

\author{
MOHAMMAD A. KAZEMI ${ }^{1}$
}

(Received 19 March 1994; revised 19 September 1994)

\begin{abstract}
In this paper we consider an optimal control problem governed by a system of nonlinear hyperbolic partial differential equations with deviating argument, Darboux-type boundary conditions and terminal state inequality constraints. The control variables are assumed to be measurable and the state variables are assumed to belong to a Sobolev space. We derive an integral representation of the increments of the functionals involved, and using separation theorems of functional analysis, obtain necessary conditions for optimality in the form of a Pontryagin maximum principle. The approach presented here applies equally well to other nonlinear constrained distributed parameters with deviating argument.
\end{abstract}

\section{Introduction}

The optimal control of dynamic systems governed by partial differential equations has been studied extensively in the literature (see [1], [2], [4], [7], [10], [17-19]). However, in many applications such as transport processes, economic systems, population models, etc., the behavior of the state may depend upon its past history. Such processes are usually represented by difference-differential equations. In addition, the majority of thermal processes, and processes in which the signal is transmitted through long electrical hydraulic lines, exhibit delays distributed along the entire length of the spatial coordinate. Processes of this type are often described by partial differential equations with delays. For specific examples of such systems with delays as well as a rather complete list of references on the development of the theory of optimal control of systems with time delays, we refer the reader to [3]. Other mathematical models associated with the control of distributed parameter systems with simple time delays, appearing in the state equations or boundary conditions have been studied in the literature (see [5], [9], [10], [12], [14], [16]). We remark that the works reported in these references exclude any type of constraints on the state variables.

\footnotetext{
'Department of Mathematics, University of North Carolina at Charlotte, Charlotte, North Carolina 28223. (C) Australian Mathematical Society, 1996, Serial-fee code 0334-2700/96
} 
One of the first steps toward solving optimal control problems with a general deviating argument is to obtain necessary conditions for optimality. Such conditions provide a source of numerical methods for computing the optimal control. In this paper we present an approach to (first order) necessary conditions for optimality in systems described by nonlinear partial differential equations with deviating argument, including terminal state inequality constraints. We explain our approach using Darboux hyperbolic partial differential equations, and obtain new results which in fact generalize the results of [5], [10], [11].

The paper is organized as follows. In Section 2, we formulate the optimal control problem and discuss the existence and uniqueness of the solution for the governing state equations. In Section 3, the adjoint equations are introduced and the existence of their solutions is established. In Section 4 we derive the increment formula for the functionals and give bounds for the remainders. Section 5 includes our necessary conditions. In Section 6, we consider more general objective functions and generalize the results of Section 5. Finally, in Section 7, we derive, under further regularity assumptions, a simplified version of results in Section 5.

\section{Problem formulation}

In this section we first describe the system and basic assumptions, and then formulate the optimal control problem. In the rectangle

$$
G:=\left\{(x, t) \in \mathbb{R}^{2} \mid x_{0} \leq x \leq X, t_{0} \leq t \leq T\right\},
$$

we consider the system

$$
\begin{gathered}
z_{x t}(x, t)=f\left(x, t, z(x, t), z(x, \sigma(t)), z_{x}(x, t), z_{x}(x, \sigma(t)),\right. \\
\left.z_{t}(x, t), z_{t}(x, \sigma(t)), u(x, t)\right), \quad \text { for a.e. }(x, t) \in G \\
z(x, t)=\phi(x, t), \quad(x, t) \in\left[x_{0}, X\right] \times\left[\sigma\left(t_{0}\right), T\right] \\
z\left(x_{0}, t\right)=\psi(t), \quad t \in\left[t_{0}, T\right],
\end{gathered}
$$

where $z(x, t)=\left(z_{1}(x, t), \ldots, z_{n}(x, t)\right)$ is the state, $u(x, t)=\left(u_{1}(x, t), \ldots, u_{m}(x, t)\right)$ is the control and $\sigma(t)$ is an absolutely continuous function satisfying $\sigma(t) \leq t$, $d \sigma(t) / d t>0$. The inverse of $\sigma(t)$ is denoted by $\gamma(t)$.

Let $U$ be a compact, convex set in $\mathbb{R}^{m}$. A measurable function $u: G \rightarrow \mathbb{R}^{m}$ is called an admissible control if $u \in U$ a.e. on $G$. We denote by $\Omega$ the class of admissible controls. For each $u \in \Omega$, a function $z:=z(u)$ in $W_{p}^{1}(G), p \in[1, \infty]$, is said to be a solution of (2.1)-(2.2) if it satisfies (2.1) a.e. on $\mathrm{G}$ and the boundary conditions (2.2) everywhere in the corresponding domain of definition. We require the following assumptions regarding the existence and uniqueness of the solution of (2.1)-(2.2). 
$\left(\mathrm{A}_{1}\right)$ The functions $\phi(x, t)$ and $\psi(t)$ are absolutely continuous on their respective domains with $\phi_{x}, \phi_{t}$ and $\psi_{t}$ belonging to $L_{p}$, for some $p \in[1, \infty]$. Furthermore, $\phi\left(x_{0}, t_{0}\right)=\psi\left(t_{0}\right)$.

$\left(\mathrm{A}_{2}\right)$ The $n$-vector function $f\left(x, t, z_{1}, \zeta_{1}, z_{2}, \zeta_{2}, z_{3}, \zeta_{3}, u\right)$ defined on $G \times \mathbb{R}^{6 n} \times U$ is measurable on $G$ for fixed $\left(z_{1}, \zeta_{1}, z_{2}, \zeta_{2}, z_{3}, \zeta_{3}\right)$, and is continuous on $U$ for fixed $\left(x, t, z_{1}, \zeta_{1}, z_{2}, \zeta_{2}, z_{3}, \zeta_{3}\right)$. Furthermore, for each $u \in \Omega$, the function $s(x, t):=f(x, t, 0,0,0,0,0,0, u(x, t))$ belongs to $L_{p}\left(G, \mathbb{R}^{n}\right)$ with $p$ as in $\left(\mathrm{A}_{1}\right)$.

$\left(\mathrm{A}_{3}\right)$ The components $f_{i}$ of $f=\left(f_{1}, \ldots, f_{n}\right)$ are continuously differentiable functions of $\left(z_{1}, \zeta_{1}, z_{2}, \zeta_{2}, z_{3}, \zeta_{3}\right)$ for fixed $(x, t, u)$.

(A) There is a function $K_{1}(x, t, u)$, with $K_{1}(x, t, u(x, t)) \in L_{\infty}(G)$ for $u \in \Omega$, such that for $i=1, \ldots, n ; j=1,2,3$,

$$
\left|\frac{\partial f_{i}}{\partial z_{j}}(x, t, Z, u)\right|,\left|\frac{\partial f_{i}}{\partial \zeta_{j}}(x, t, Z, u)\right| \leq K_{1}(x, t, u),
$$

where $Z(x, t):=\left(z(x, t), z(x, \sigma(t)), z_{x}(x, t), z_{x}(x, \sigma(t)), z_{t}(x, t), z_{t}(x, \sigma(t))\right.$.

Let $u \in \Omega$ be given. To prove the existence of a unique solution $z:=z(u)$ to (2.1)-(2.2), we first note that over the subrectangle $\left[x_{0}, X\right] \times\left[t_{0}, \gamma\left(t_{0}\right)\right]$, the functions $z(x, \sigma(t)), z_{x}(x, \sigma(t))$, and $z_{t}(x, \sigma(t))$ are known in terms of the boundary data $\phi(x, t)$. Thus we can (as in [1], [11]) prove the existence of a unique solution to (2.1)-(2.2) over $\left[x_{0}, X\right] \times\left[t_{0}, \gamma\left(t_{0}\right)\right]$. Next, using $z\left(x, \gamma\left(t_{0}\right)\right)$ as a boundary data, we apply the same argument to extend the solution further to $\left[x_{0}, X\right] \times\left[\gamma\left(t_{0}\right), \gamma\left(\gamma\left(t_{0}\right)\right)\right]$, and so on until the whole rectangle is covered. Thus for a given $u \in \Omega$, the system (2.1)-(2.2) has a unique solution $z(x, t):=z(u)(x, t)$. Furthermore, the estimates of Theorems (3.1)-(3.2) of [11] still hold in our case, as can be easily seen. See also [1], Section 4.3.

Now let the performance of the control process be estimated at $x=X, t=T$ by the functionals

$$
J_{k}(u):=g_{k}(z(X, T)), \quad k=0,1, \ldots, r .
$$

We are concerned with the following optimal control problem.

Find $\hat{u} \in \Omega$ and the corresponding $\hat{z}:=z(\hat{u})$, from (2.1)-(2.2), such that

$$
J_{k}(\hat{u}) \leq 0 \text {, for } k=1,2, \ldots, r \text {, and } J_{0}(\hat{u}) \leq J_{0}(u) \text { for all } u \in \Omega \text {. }
$$

We will refer to such a $u$ as an optimal control.

We require the following assumption regarding the values $g_{k}$.

(A) The functions $g_{k}: \mathbb{R}^{n} \rightarrow \mathbb{R}, k=0,1, \ldots, r$ are twice continuously differentiable. 


\section{The adjoint equations}

Define

$$
\begin{aligned}
H^{(k)}\left(x, t, z, \zeta, z_{x}, \zeta_{x}, z_{t}, \zeta_{t}, u, \lambda^{(k)}, \mu^{(k)}, p^{(k)}, q^{(k)}\right):= \\
\lambda^{(k)} \cdot z_{k}+\mu^{(k)} \cdot z_{t}+\left(p^{(k)}+q^{(k)}\right) \cdot f\left(x, t, z, \zeta, z_{x}, \zeta_{x}, z_{t}, \zeta_{t}, u\right)
\end{aligned}
$$

NOTATION. Let $\hat{u} \in \Omega$ and $\hat{z}:=z(\hat{u})$ be an admissible pair for problem (P). In what follows, for simplicity of notation, we denote by $\hat{H}^{(k)}(x, t)$, the value of $H^{(k)}$ at $\left(x, t, \hat{z}(x, t), \hat{z}(x, \sigma(t)), \hat{z}_{x}(x, t), \hat{z}_{x}(x, \sigma(t)), \hat{z}_{t}(x, t), \hat{z}_{t}(x, \sigma(t)), \hat{u}(x, t), \lambda^{(k)}(x\right.$, $\left.t), \mu^{(k)}(x, t), \hat{p}^{(k)}(x, t), \hat{q}^{(k)}(x, t)\right)$. Similarly, $\hat{H}^{(k)}(x, \gamma(t))$ denotes the value of $H^{(k)}$ at $\left(x, \gamma(t), z(x, \gamma(t)), z(x, t), z_{x}(x, \gamma(t)), z_{x}(x, t), z_{t}(x, \gamma(t)), z_{t}(x, t), \hat{u}(x, \gamma(t))\right.$, $\hat{\lambda}^{(k)}(x, \gamma(t)), \hat{\mu}^{(k)}(x, \gamma(t)), \hat{q}^{(k)}(x, \gamma(t))$. Also note that $\hat{\lambda}^{(k)}(x, t):=\lambda^{(k)}(\hat{u})(x, t)$, etc. We now define the adjoint equations (or the linear conjugate problem) by the following system of linear partial differential equations and boundary conditions.

$$
\begin{aligned}
\hat{\lambda}_{x}^{(k)}+\hat{\mu}_{t}^{(k)} & =-\hat{H}_{z}^{(k)}(x, t)-\dot{\gamma}(t) \hat{H}_{\zeta}^{(k)}(x, \gamma(t)), & & (x, t) \in\left[x_{0}, X\right] \times\left[t_{0}, \sigma(T)\right] \\
\hat{\lambda}_{x}^{(k)}+\hat{\mu}_{t}^{(k)} & =-\hat{H}_{z}^{(k)}(x, t), & & (x, t) \in\left[x_{0}, X\right] \times[\sigma(T), T] \\
\hat{p}_{x}^{(k)} & =-\hat{H}_{z_{t}}^{(k)}(x, t)-\dot{\gamma}(t) \hat{H}_{\zeta_{t}}^{(k)}(x, \gamma(t)), & & (x, t) \in\left[x_{0}, X\right] \times\left[t_{0}, \sigma(T)\right] \\
\hat{p}_{x}^{(k)} & =-\hat{H}_{z_{t}}^{(k)}(x, t), & & (x, t) \in\left[x_{0}, X\right] \times[\sigma(T), T] \\
\hat{q}_{t}^{(k)} & =-\hat{H}_{z_{x}}^{(k)}(x, t)-\dot{\gamma}(t) \hat{H}_{\zeta_{x}}^{(k)}(x, \gamma(t)), & & (x, t) \in\left[x_{0}, X\right] \times\left[t_{0}, \sigma(T)\right] \\
\hat{q}_{t}^{(k)} & =-\hat{H}_{z_{x}}^{(k)}(x, t), & & (x, t) \in\left[x_{0}, X\right] \times[\sigma(T), T] \\
\hat{\lambda}^{(k)}(X, t) & =\hat{\mu}^{(k)}(x, T)=0, & & t \in\left[t_{0}, T\right], x \in\left[x_{0}, X\right] \\
p^{(k)}(X, t) & =q^{(k)}(x, T)=g_{k z}(\hat{z}(X, T)) / 2, & & t \in\left[t_{0}, T\right], x \in\left[x_{0}, X\right] .
\end{aligned}
$$

We have the following result.

THEOREM 3.1. Let $\hat{u} \in \Omega$ and let $\hat{z}$ be the corresponding solution of (2.1)-(2.2). Then there exist solutions $\hat{\lambda}^{(k)}, \hat{\mu}^{(k)}, \hat{p}^{(k)}, \hat{q}^{(k)}$ in $\left[L_{\infty}(G)\right]^{n}$ with $\hat{\lambda}^{(k)}, \hat{p}^{(k)}$ absolutely continuous with respect to $x$, and $\hat{\mu}^{(k)}, \hat{q}^{(k)}$ absolutely continuous with respect to $t$, satisfying (3.1)-(3.5).

PROOF. We first consider the system in the subrectangle $\left[x_{0}, X\right] \times[\sigma(T), T]$ : 


$$
\begin{gathered}
\hat{\lambda}_{x}^{(k)}+\hat{\mu}_{t}^{(k)}=-\hat{H}_{z}^{(k)}(x, t), \\
\hat{q}_{x}^{(k)}=-\hat{H}_{z_{x}}^{(k)}(x, t), \\
\hat{p}_{x}^{(k)}=-\hat{H}_{z_{t}}^{(k)}(x, t), \\
\hat{\lambda}^{(k)}(X, t)=\hat{\mu}^{(k)}(x, T)=0, \\
\hat{p}^{(k)}(X, t)=\hat{q}^{(k)}(x, T)=\frac{1}{2} g_{k z}(\hat{z}(X, T)) .
\end{gathered}
$$

This system is equivalent to a system of two-dimensional Volterra integral equations. To see this, let $\theta^{(k)}(x, t):=p^{(k)}(x, t)+q^{(k)}(x, t)$ and formally integrate (3.6) as follows.

$$
\begin{aligned}
\lambda^{(k)}(x, t)= & \int_{X}^{x} \lambda_{x}^{(k)}(\alpha, t) d \alpha \\
\mu^{(k)}(x, t)= & -\int_{T}^{t}\left[\lambda_{x}^{(k)}(x, \beta)+\hat{f}_{z}(x, \beta)^{\top} \theta^{(k)}(x, \beta)\right] d \beta \\
p^{(k)}(x, t)= & \frac{1}{2} g_{k z}(\hat{z}(X, T))+\int_{X}^{x} \int_{T}^{t}\left[\lambda_{x}^{(k)}(\alpha, \beta)++\hat{f}(\alpha, \beta)^{\top} \omega^{(k)}(\alpha, \beta)\right] d \alpha d \beta \\
& -\int_{X}^{x} \hat{f}_{z_{t}}(\alpha, t)^{\top} \theta^{(k)}(\alpha, t) d \alpha \\
q^{(k)}(x, t)= & \frac{1}{2} g_{k z}(\hat{z}(X, T))-\int_{T}^{t} \int_{X}^{x} \lambda_{x}^{(k)}(\alpha, \beta) d \alpha d \beta-\int_{T}^{t} \hat{f}_{z_{x}}(x, \beta)^{\top} \theta^{(k)}(x, \beta) d \beta
\end{aligned}
$$

Thus $\theta^{(k)}$ satisfies the integral equation $I \theta=\theta$, where

$$
\begin{aligned}
(I \theta)(x, t):= & g_{z}(\hat{z}(X, T))+\int_{x}^{X} \int_{t}^{T} \hat{f}_{z}(\alpha, \beta)^{\top} \theta(\alpha, \beta) d \alpha d \beta \\
& +\int_{t}^{T} \hat{f}_{z_{x}}(x, \beta)^{\top} \theta(x, \beta) d \beta+\int_{x}^{X} \hat{f}_{z_{t}}(\alpha, t)^{\top} \theta(\alpha, t) d \alpha .
\end{aligned}
$$

The integral equation $I \theta=\theta$ can be shown to have a unique solution $\theta \in\left[L_{\infty}(G)\right]^{n}$ (see [1]). Having $\theta^{(k)}$, we can find from (3.6) $\mu^{(k)}, p^{(k)}$ and $q^{(k)}$ in terms of $\lambda^{(k)}$ and $\theta^{(k)}$ for $(x, t) \in\left[x_{0}, X\right] \times[\sigma(T), T]$. Note therefore that $\hat{H}_{\zeta}^{(k)}(x, \gamma(t)) \hat{H}_{\zeta_{x}}^{(k)}(x, \gamma(t))$, and $\hat{H}_{\zeta_{t}}^{(k)}(x, \gamma(t))$, are known over the subrectangle $\left[x_{0}, X\right] \times[\sigma(\sigma(T)), \sigma(T)]$. Thus by repeating the above argument the solution is extended to $\left[x_{0}, X\right] \times[\sigma(\sigma(T)), \sigma(T)]$, and so on until the whole rectangle is covered. This completes the proof of the theorem.

REMARK 3.1. Note that since $\hat{\theta}^{(k)}:=\hat{p}^{(k)}+\hat{q}^{(k)}$ is uniquely determined as the fixed point of $I$, for different choices of $\hat{\lambda}^{(k)}$ we will get the same $\hat{p}^{(k)}+\hat{q}^{(k)}$. 


\section{The increment formula}

In this section we derive integral representations for the increments $J_{k}(u)-$ $J_{k}(\hat{u}), k=0,1, \ldots, r$, where $u, \hat{u} \in \Omega$. The corresponding states are denoted by $\hat{z}:=z(\hat{u})$ and $z:=z(u)$. To simplify the exposition, we use the notation

$$
\begin{gathered}
\hat{g}_{z}:=\frac{\partial g}{\partial z}(\hat{z}(X, T)), \\
\Delta z:=z-\hat{z}, \\
\hat{f}(x, t):=f(x, t, \hat{z}(x, t), \hat{z}(x, \sigma(t)), \ldots, \hat{u}(x, t)), \\
\Delta_{u, \hat{u}} f(x, t):=f\left(x, t, \hat{z}(x, t), \hat{z}(x, \sigma(t)), \ldots, \hat{z}_{t}(x, \sigma(t)), u(x, t)\right)-\hat{f}(x, t) .
\end{gathered}
$$

Similar notation will be used when we write $\Delta_{u, \hat{u}} H^{(k)}(x, t)$. We have

$$
\begin{aligned}
J_{k}(u)-J_{k}(\hat{u}) & =\hat{g}_{k z} \cdot \Delta z(X, T)+o(\|z\|) \\
& =\frac{1}{2} \hat{g}_{k z} \cdot \Delta z(X, T)+\frac{1}{2} \hat{g}_{k z} \cdot \Delta z(X, T)+o(\|z\|) \\
& =\int_{x_{0}}^{X} \frac{1}{2} \hat{g}_{k z} \cdot \Delta z_{x}(x, T) d x+\int_{t_{0}}^{T} \frac{1}{2} \hat{g}_{k z} \cdot \Delta z_{t}(X, t) d t+o(\|z\|) .
\end{aligned}
$$

Using the boundary conditions (3.4)-(3.5) along with integration by parts, we continue as follows.

$$
\begin{aligned}
J_{k}(u)-J_{k}(\hat{u})= & \int_{x_{0}}^{X} q^{(k)}(x, T) \cdot \Delta z_{x}(x, T) d x \\
& \quad+\int_{t_{0}}^{T} p^{(k)}(X, t) \cdot \Delta z_{t}(X, t) d t+o(\|\Delta z\|) \\
= & \iint_{G}\left(q_{t}^{(k)} \cdot \Delta z_{x}+p^{(k)} \cdot \Delta z_{x t}\right) d x d t \\
& \quad+\iint_{G}\left(p_{x}^{(k)} \cdot \Delta z_{t}+p^{(k)} \cdot \Delta z_{x t}\right) d x d t \\
= & \iint_{G}\left(q_{t}^{(k)} \cdot \Delta z_{x}+p^{(k)} \cdot \Delta z_{x t}\right) d x d t \\
& \quad+\iint_{G}\left(p_{x}^{(k)}+q^{(k)}\right) \cdot \Delta z_{x t} d x d t+o(\|\Delta z\|) .
\end{aligned}
$$

Substitution from (3.2)-(3.3) into the first integral on the right-hand side of (4.1) yields 


$$
\begin{aligned}
& \iint_{G}\left(q_{t}^{(k)} \cdot \Delta z_{x}+p_{x}^{(k)} \cdot z_{t}\right) d x d t \\
&=-\int_{x_{0}}^{X} \int_{t_{0}}^{\sigma(T)}\left[\hat{H}_{z_{x}}^{(k)}(x, t)+\dot{\gamma}(t) \hat{H}_{\zeta_{x}}^{(k)}(x, \gamma(t))\right] \Delta z_{x}(x, t) d x d t \\
&-\int_{x_{0}}^{X} \int_{\sigma(T)}^{T} \hat{H}_{z_{x}}^{(k)}(x, t) \Delta z_{x}(x, t) d x d t \\
&-\int_{x_{0}}^{X} \int_{t_{0}}^{\sigma(T)}\left[\hat{H}_{z_{t}}^{(k)}(x, t)+\dot{\gamma}(t) \hat{H}_{\zeta_{t}}^{(k)}(x, \gamma(t))\right] \Delta z_{t}(x, t) d x d t \\
&-\int_{x_{0}}^{X} \int_{\sigma(T)}^{T} \hat{H}_{z_{t}}^{(k)}(x, t) \Delta z_{t}(x, t) d x d t \\
&=\left.-\int_{\int_{G}} \int_{z_{x}}^{(k)}(x, t) \Delta z_{x}(x, t)+\hat{H}_{z_{t}}^{(k)}(x, t) \Delta z_{t}(x, t)\right] d x d t \\
&-\int_{z_{0}}^{X} \int_{t_{0}}^{\sigma(T)}\left[\dot{\gamma}^{(t)}\left(\hat{H}_{\zeta_{t}}^{(k)}(x, \gamma(t)) \Delta z_{x}(x, t)+\dot{\gamma}(t) \hat{H}_{\zeta_{t}}^{(k)}(x, \gamma(t)) \Delta z_{t}(x, t)\right] d x d t\right.
\end{aligned}
$$

On making the change of variable $\tau=\gamma(t), t=\sigma(\tau)$, the second integral in the last expression becomes

$$
\int_{x_{0}}^{X} \int_{\gamma\left(t_{0}\right)}^{T}\left[\hat{H}_{\zeta_{x}}^{(k)}(x, \tau) \Delta z_{x}(x, \sigma(\tau))+\hat{H}_{\zeta_{t}}^{(k)}(x, \tau) \Delta z_{t}(x, \sigma(\tau))\right] d x d t .
$$

Noting that $\Delta z(x, t) \equiv 0$ for $(x, t) \in\left[x_{0}, X\right] \times\left[\sigma\left(t_{0}\right), t_{0}\right]$, and hence $\Delta z_{x}(x, t)=$ $\Delta z_{t}(x, t) \equiv 0$ for $(x, t) \in\left[x_{0}, X\right] \times\left[\sigma\left(t_{0}\right), t_{0}\right]$, we see that $\Delta z_{x}(x, \sigma(\tau))=\Delta z_{t}(x$, $\sigma(\tau))=0$ for $\tau \in\left[t_{0}, \gamma\left(t_{0}\right)\right]$. Thus we conclude that

$$
\begin{aligned}
& \iint_{G}\left[q_{t}^{(k)} \cdot \Delta z_{x}+p_{x}^{(k)} \cdot \Delta z_{t}\right] d x d t= \\
& -\iint_{G}\left[\hat{H}_{z_{x}}^{(k)}(x, t) \cdot \Delta z_{x}(x, t)+\hat{H}_{\zeta_{x}}^{(k)}(x, t) \Delta z_{x}(x, \sigma(t))\right. \\
& \left.\quad+\hat{H}_{z_{t}}^{(k)}(x, t) \cdot \Delta z_{t}(x, t)+\hat{H}_{\xi_{t}}^{(k)}(x, t) \cdot \Delta z_{t}(x, \sigma(t))\right] d x d t .
\end{aligned}
$$

To simplify the second integral in (4.1), we write

$$
\begin{aligned}
\Delta z_{x t}= & f(x, t, z(x, t), \ldots, u(x, t))-f(x, t, \hat{z}(x, t), \ldots, \hat{u}(x, t)) \\
= & \Delta_{u, \hat{u}} f(x, t)+f\left(x, t, z(x, t), \ldots, z_{t}(x, \sigma(t)), u(x, t)\right) \\
& -f\left(x, t, \hat{z}(x, t), \ldots, \hat{z}_{t}(x, \sigma(t)), u(x, t)\right) .
\end{aligned}
$$

If we apply the mean-value theorem, the last relation yields

$$
\Delta z_{x t}=\Delta_{u, \hat{u}} f(x, t)+f_{z}(x, t, \tilde{Z}(x, t), u(x, t)) \Delta Z,
$$


where $Z(x, t):=\left(z(x, t), z(x, \sigma(t)), z_{x}(x, \sigma(t)), z_{t}(x, t), z_{t}(x, \sigma(t))\right)$ and $\tilde{Z}:=Z+$ $\theta(x, t)(Z-\hat{Z})$ for some function $\theta(x, t), 0<\theta<1$. Thus the second integral in (4.1) takes the form

$$
\begin{aligned}
& \iint_{G}\left(p^{(k)}+q^{(k)}\right) \cdot \Delta z_{x t}= \\
& \quad \iint_{G}\left(p^{(k)}+q^{k}\right) \cdot \Delta_{u, \hat{u}} f(x, t) d x d t+\iint_{G}\left(p^{(k)}+q^{(k)}\right)^{\top} f_{Z}(x, t) \Delta Z d x d t \\
& \quad+\iint_{G}\left(p^{(k)}+q^{(k)}\right)^{\top}\left[f_{Z}(x, t, \tilde{Z}(x, t), u(x, t))-\hat{f}_{z}(x, t)\right] \Delta Z d x d t .
\end{aligned}
$$

Substitution of (4.2)-(4.3) into (4.1) yields, for $k=0,1, \ldots, r$,

$$
\begin{aligned}
J_{k}(u) & -J_{k}(\hat{u})= \\
& \iint_{G} \Delta_{u, \hat{u}} H^{(k)}(x, t) d x d t \\
& +\iint_{G}\left[\lambda^{(k)} \cdot \Delta z_{x}+\mu^{(k)} \cdot \Delta z_{t}+\left(p^{(k)}+q^{(k)}\right)^{\top} \hat{f}_{z}^{(k)} \Delta z\right] d x d t \\
& +\iint_{G}\left(p^{(k)}+q^{(k)}\right)^{\top}\left[\hat{f}_{z}(x, t, \tilde{Z}(x, t), u(x, t))-\hat{f}_{z}(x, t)\right] \Delta Z d x d t \\
& +o(\|\Delta z\|) .
\end{aligned}
$$

Note from (3.1)-(3.4) and (2.2) that the second integral in the above expression vanishes. We have finally arrived at the integral representation

$$
J_{k}(u)-J_{k}(\hat{u})=\iint_{G} \Delta_{u, \hat{u}} H^{(k)}(x, t) d x d t+\eta_{k},
$$

where

$\eta_{k}:=\iint_{G}\left(p^{(k)}+q^{(k)}\right)^{\top}\left[f_{Z}(x, t, \tilde{Z}(x, t), u(x, t))-\hat{f}_{Z}(x, t)\right] \Delta Z d x d t+o(\|\Delta x\|)$ is the remainder term, to be estimated below. To estimate $\eta_{k}$, we require the following assumptions.

(A6) There exists a nonnegative real-valued function $M(x, t, u)$ defined on $G \times U$ with $M(x, t, u(x, t)) \in L_{4}(G)$ for $u \in \Omega$; and for $p \in[1, \infty)$, there exists a constant $K_{2} \geq 0$ such that

$$
\left|f\left(x, t, z_{1}, \zeta_{1}, z_{2}, \zeta_{2}, z_{3}, \zeta_{3}, u\right)\right| \leq M(x, t, u)+K_{2}\left\{\sum_{i=1}^{3}\left|z_{i}\right|+\left|\zeta_{i}\right|\right\}^{p / 4}
$$

for all $\left(x, t, z_{1}, \zeta_{1}, z_{2}, \zeta_{2}, z_{3}, \zeta_{3}, u\right) \in G \times \mathbb{R}^{6 n} \times U$.

If $p=\infty$, then we require $|f| \leq M(x, t, u)+K_{2}\left(\sum_{i=1}^{3}\left|z_{i}\right|+\left|\zeta_{i}\right|\right)$. 
$\left(\mathrm{A}_{7}\right)$ The partial derivatives $f_{z_{i}}, f_{\zeta_{i}}, i=1,2,3$, satisfy a Lipschitz condition with respect to $\left(z_{1}, \zeta_{1}, z_{2}, \zeta_{2}, z_{3}, \zeta_{3}\right)$.

Now let $\left(x_{0}, t_{0}\right)$ be an interior point of $G$ at a distance $\delta>0$ from $\partial G$. For $0<\delta<\delta_{0}$ denote by $G_{\delta}$ the square

$$
G_{\delta}:=\left\{(x, t) \in G \mid x_{0} \leq x<x_{0}+\delta, t_{0} \leq t<t_{0}+\delta\right\} .
$$

For $\hat{u} \in \Omega$ and $v$ an arbitrary element of $U$, define the (admissible) control variation

$$
u_{\delta}(x, t):= \begin{cases}\hat{u}(x, t), & (x, t) \in G \backslash G_{\delta} \\ v, & (x, t) \in G_{\delta} .\end{cases}
$$

We have the following.

LEMMA 4.1. Suppose that assumptions $\left(A_{1}\right)-\left(A_{7}\right)$ hold. Then there exists a constant $K_{3}$ independent of $\hat{u}, v$ and such that the remainder term $\eta_{k}, 0 \leq k \leq r$, in (4.4) satisfies the estimate

$$
\left|\eta_{k}\right| \leq K_{3} \delta^{2} B(\hat{u}, v, \delta)
$$

where

$$
\begin{aligned}
B(\hat{u}, v, \delta):= & \iint_{G}\left|\Delta_{u_{\delta}, \hat{u}} f(x, t)\right|^{2} d x d t+\left(\iint_{G}\left|\Delta_{u_{s}, \hat{u}} f(x, t)\right|^{2} d x d t\right)^{1 / 2} \\
& +\left(\iint_{G}\left|\Delta_{u_{\delta}, \hat{u}} f(x, t)\right|^{4} d x d t\right)^{1 / 2}
\end{aligned}
$$

and $B(\hat{u}, v, \delta) \rightarrow 0$ as $\delta \rightarrow 0$.

PROOF. The estimate (4.6) follows from the same line of analysis as in [1] or [11]. We omit the details. Since, by $\left(A_{6}\right),\left|\Delta_{u_{g}, \hat{u}} f(x, t)\right| \in L_{4}(G)$ and $G$ is bounded, it follows that $B(\hat{u}, v, \delta) \rightarrow 0$ as $\delta \rightarrow 0$.

From (4.4) and Lemma 4.1, we have therefore obtained the following integral representation for the increment of $J$.

THEOREM 4.1. Suppose that assumptions $\left(A_{1}\right)-\left(A_{6}\right)$ hold. Then with $u:=u_{\delta}$ defined as in (4.5),

$$
J_{k}\left(u_{6}\right)-J_{k}(\hat{u})=\iint_{G} \Delta_{u_{b}, u} H^{(k)}(x, t) d x d t+\eta_{k},
$$

where $\eta_{k}$ satisfies (4.6). 


\section{Necessary conditions}

In this section we state and prove necessary conditions for optimality for problem $(\mathrm{P})$, in the form of a Pontryagin maximum principle. We begin by rewriting (4.7) as

$$
\begin{gathered}
J_{k}\left(u_{\delta}\right)-J_{k}(\hat{u})=\delta^{2} \Delta_{v, \hat{u}} H^{(k)}\left(x_{0}, t_{0}\right)+o\left(\delta^{2}\right), \\
k=0,1, \ldots, r ; \quad \text { for a.e. }\left(x_{0}, t_{0}\right) \in \operatorname{Int} G .
\end{gathered}
$$

This of course follows by standard arguments involving the Lebesgue differentiation theorem (see [1] or [15]). Here $\Delta_{v, \hat{u}} H^{(k)}\left(x_{0}, t_{0}\right)$ denotes the difference

$$
\begin{aligned}
& H^{(k)}\left(x_{0}, t_{0}, \hat{Z}\left(x_{0}, t_{0}\right), v, \lambda^{(k)}\left(x_{0}, t_{0}\right), \mu^{(k)}\left(x_{0}, t_{0}\right), p^{(k)}\left(x_{0}, t_{0}\right), q^{(k)}\left(x_{0}, t_{0}\right)\right) \\
& -H^{(k)}\left(x_{0}, t_{0}, \hat{Z}\left(x_{0}, t_{0}\right), \hat{u}\left(x_{0}, t_{0}\right), \lambda^{(k)}\left(x_{0}, t_{0}\right), \mu^{(k)}\left(x_{0}, t_{0}\right), p^{(k)}\left(x_{0}, t_{0}\right), q^{(k)}\left(x_{0}, t_{0}\right)\right) .
\end{aligned}
$$

Next let $(x, t) \in \tilde{G}$, where $\tilde{G}$ denotes the intersection of the interior of $G$ and the Lebesgue point of $\Delta_{v, \hat{u}}:=H^{(k)}$, and define

$$
\begin{gathered}
a_{k}(x, t ; v, \hat{u}):=\Delta_{v, \hat{u}} H^{(k)}(x, t), \quad 0 \leq k \leq r \\
a(x, t ; v, \hat{u})=\left(a_{0}(x, t ; v, \hat{u}), \ldots, a_{r}(x, t ; v, \hat{u})\right), \\
A:=\left\{a(x, t ; v, \hat{u}) \in \mathbb{R}^{1+r} \mid v \in U, \hat{u} \in \Omega,(x, t) \in \tilde{G}\right\}, \\
B:=\left\{b=\left(b_{0}, b_{1}, \ldots, b_{r}\right) \in \mathbb{R}^{1+r} \mid b_{k}<0 \text { for } k \in I\right\} .
\end{gathered}
$$

THEOREM 5.1. Let $\hat{u} \in \Omega$ be an optimal control for problem $(P)$, and suppose that assumptions $\left(A_{1}\right)-\left(A_{7}\right)$ are satisfied. Then the set $B$ and the convex hull $\mathrm{CH}(A)$ of the set $A$ have no common points, that is, $B \cap \mathrm{CH}(A)=\emptyset$.

Proof. Suppose, on the contrary, that there is an element $c \in \mathbb{R}^{1+r}$ such that $c \in$ $B \cap \mathrm{CH}(A)$. Then by the definition of the convex hull, there are points $\left(x_{i}, t_{i}\right) \in \tilde{G}$ and $v_{i} \in U$, and $\theta_{i}, i=1, \ldots, \ell$, such that

$$
c=\sum_{i=1}^{\ell} \theta_{i} a\left(x_{i}, t_{i} ; v_{i}, \hat{u}\right), \quad \theta_{i}>0, \sum_{i=1}^{\ell} \theta_{i}=1 .
$$

Moreover, since $c \in B$, its components $c_{j}$ satisfy

$$
c_{j}=\sum_{i=1}^{\ell} \theta_{i} a_{j}\left(x_{i}, t_{i} ; v_{i}, \hat{u}\right)<0 \quad \text { for } j \in I .
$$

Below, using the set of parameters $\left\{x_{i}, t_{i} ; v_{i}, \theta_{i}\right\}, 1 \leq i \leq \ell$, we construct a special variation $u_{\delta}$ of the optimal control $\hat{u}$ which is admissible, satisfies $J_{k}\left(u_{\delta}\right) \leq 0$ for $k=1, \ldots, r$, and $J_{0}\left(u_{\delta}\right)<J_{0}(\hat{u})$. This contradiction proves the theorem. 
If $\left(x_{p}, t_{p}\right)$ is an isolated point of the set $\left\{x_{i}, t_{i}\right\}, 1 \leq i \leq \ell$, that is, if $\left(x_{p}, t_{p}\right) \neq\left(x_{i}, t_{i}\right)$ for $p_{i}$, we set $G_{\delta}^{(p)}:=\left\{(x, t) \in G \mid x_{p} \leq x<x_{p}+\delta \theta_{p}, t_{p} \leq t<t_{p}+\delta\right\}$. If

$$
\left(x_{p_{1}}, t_{p_{1}}\right)=\cdots=\left(x_{p_{v}}, t_{p_{v}}\right), \quad 1 \leq p_{1}<\cdots<p_{v} \leq \ell,
$$

then for $m=1, \ldots, v$ set

$$
G_{\delta}^{\left(p_{m}\right)}:=\left\{(x, t) \in G \mid x_{p_{1}}+\delta \sum_{i=1}^{m-1} \theta_{p_{i}} \leq x<x_{p_{1}}+\delta \sum_{i=1}^{m} \theta_{p_{i}}, t_{p_{1}} \leq t<t_{p_{1}}+\delta\right\} .
$$

We note that in either case, the measure (area) of $G_{\delta}^{(p)}$ is $\delta^{2} \theta_{p}$. We choose $\delta>0$ sufficiently small so that the rectangles $G_{\delta}^{(p)}$ are pairwise disjoint. For each $i=$ $1, \ldots, \ell$, we now set

$$
u_{\delta}^{(i)}(x, t):= \begin{cases}\hat{u}(x, t), & \text { for }(x, t) \in G \backslash G_{i} \\ v_{i}, & \text { for }(x, t) \in G_{i},\end{cases}
$$

and define the convex combination $u_{\delta}(x, t):=\sum_{i=1}^{\ell} \theta_{i} u_{\delta}^{(i)}(x, t), \quad(x, t) \in G$. By the convexity of the set $U, u_{\delta}(x, t) \in \Omega$. Now a modification of the proof of Lemma 4.3.2 of [1] shows that the estimate (4.6) still holds for the control variation given above and we have as in (5.1),

$$
J_{k}\left(u_{\delta}\right)-J_{k}(\hat{u})=\sum_{j=1}^{\ell} \theta_{j} a_{k}\left(x_{j}, t_{j} ; v_{j}, \hat{u}\right) \delta^{2}+o\left(\delta^{2}\right) \quad k=0,1, \ldots, r .
$$

From the definition of the set $I$ and (5.5), it now follows that for small enough $\delta$,

$$
\begin{aligned}
& J_{k}\left(u_{\delta}\right)<J_{k}(\hat{u}) \leq 0, \quad k=1, \ldots, r, \\
& J_{0}\left(u_{\delta}\right)<J_{0}(\hat{u}) .
\end{aligned}
$$

This contradicts the optimality of $\hat{u}$ and the proof is complete.

REMARK 5.1. The basic idea of the above proof is well-known (see [8], [13]).

Next we state our necessary condition in the form of a pointwise maximum principle. In the following theorem $I$ denotes the set of inactive constraint indices defined above.

THEOREM 5.2. Let $\hat{u} \in \Omega$ be an optimal control for problem $(P)$, and suppose that assumptions $\left(A_{1}\right)-\left(A_{7}\right)$ are satisfied. Then there exist a nonzero vector $\chi=$ $\left(\chi_{0}, \chi_{1}, \ldots, \chi_{r}\right) \in \mathbb{R}^{1+r}$ with

$$
\chi_{k} \geq 0 \quad \text { for } k \in I ; \quad \chi_{k}=0 \text { for } k \in\{0,1, \ldots, r\} \backslash I,
$$


and a set of multipliers $(\lambda, \mu, p, q)$ in $L_{\infty}\left(G, \mathbb{R}^{4 n}\right)$ such that the maximum principle

$$
\hat{H}(x, t) \leq \hat{H}(x, t ; v) \text { for all } v \in U \text { and for a.e. }(x, t) \in G
$$

holds, where

$$
\begin{aligned}
& H\left(x, t, z, \zeta, z_{x}, \zeta_{x}, z_{t}, \zeta_{t}, u, \lambda, \mu, p, q\right):= \\
& \lambda z_{x}+\mu z_{t}(p+q) f\left(x, t, z, \zeta, z_{x}, \zeta_{x}, z_{t}, \zeta_{t}, u\right)
\end{aligned}
$$

and

$$
\begin{array}{cc}
\hat{\lambda}_{x}+\hat{\mu}_{t}=-\hat{H}_{z}(x, t)-\dot{\gamma}(t) \hat{H}_{\zeta}(x, \gamma(t)), & (x, t) \in\left[x_{0}, X\right] \times\left[t_{0}, \sigma(T)\right] \\
\hat{\lambda}_{x}+\hat{\mu}_{t}=-\hat{H}_{z}(x, t), & (x, t) \in\left[x_{0}, X\right] \times[\sigma(T), T] \\
\hat{p}_{x}=-\hat{H}_{z_{t}}(x, t)-\dot{\gamma}(t) \hat{H}_{\zeta_{t}}(x, \gamma(t)), & (x, t) \in\left[x_{0}, X\right] \times\left[t_{0}, \sigma(t)\right] \\
\hat{p}_{x}=-\hat{H}_{z_{t}}(x, t), & (x, t) \in\left[x_{0}, X\right] \times[\sigma(T), T] \\
\hat{q}_{t}=-\hat{H}_{z_{x}}(x, t)-\dot{\gamma}(t) \hat{H}_{\zeta_{x}}(x, \gamma(t)), & (x, t) \in\left[x_{0}, X\right] \times\left[t_{0}, \sigma(t)\right] \\
\hat{q}_{t}=-\hat{H}_{z_{x}}(x, t), & (x, t) \in\left[x_{0}, X\right] \times[\sigma(T), T] \\
\hat{\lambda}(X, t)=0, \quad t \in\left[t_{0}, T\right] ; \quad \hat{\mu}(x, T)=0, \quad x \in\left[x_{0}, X\right] \\
\hat{p}(X, t)=\frac{1}{2} \sum_{k=0}^{r} \chi_{k} \frac{\partial g_{k}}{\partial z}(\hat{z}(X, T)), & t \in\left[t_{0}, T\right] \\
\hat{q}(x, T)=\frac{1}{2} \sum_{k=0}^{r} \chi_{k} \frac{\partial g_{k}}{\partial z}(\hat{z}(X, T)), & x \in\left[x_{0}, X\right] .
\end{array}
$$

Proof. Since the convex sets $B$ and the convex hull $\mathrm{CH}(A)$ have empty intersections, by the standard separation theorems, there exists a nonzero vector $\chi=$ $\left(\chi_{0}, \chi_{1}, \ldots, \chi_{r}\right) \in \mathbb{R}^{1+r}$ such that

$$
\chi \cdot b \leq 0 \quad \text { for all } b \in B ; \quad \chi \cdot a \geq 0 \quad \text { for all } a \in A .
$$

By the definition of the set $B$, the first inequality in (5.14) yields

$$
\chi_{k} \geq 0 \quad \text { for } k \in I ; \quad \chi_{k}=0 \quad \text { for } k \in\{0,1, \ldots, r\} \backslash I .
$$

According to the definition of the set $A$, the second inequality in (5.14) yields

$$
\sum_{k=1}^{r} \chi_{k} \Delta_{v, \hat{u}} \hat{H}_{k}\left(x_{0}, t_{0}\right) \geq 0 \quad \text { for all } v \in U \text {, a.e. }\left(x_{0}, t_{0}\right) \in \tilde{G}
$$


Define

$$
\begin{aligned}
\lambda(x, t) & :=\sum_{k=0}^{r} \chi_{k} \lambda^{(k)}(x, t), \quad \mu(x, t):=\sum_{k=0}^{r} \chi_{k} \mu^{(k)}(x, t), \\
p(x, t) & :=\sum_{k=0}^{r} \chi_{k} p^{(k)}(x, t), \quad q(x, t):=\sum_{k=0}^{r} \chi_{k} q^{(k)}(x, t), \\
H & :=\sum_{k=0}^{r} \chi_{k} H^{(k)},
\end{aligned}
$$

where $H^{(k)}$ 's are as in Section 3. We see that relations (5.8)-(5.13) follow from (3.1)(3.6). The minimum condition (5.7) follows immediately from (5.15) and the facts that $\hat{H}$ is continuous on $U$ and that almost all $(x, t) \in G$ are regular points of $\hat{H}$. This completes the proof.

\section{More general objective functions}

Suppose that the cost functionals $J_{k}$ are given by

$$
\begin{aligned}
J_{k}(u):= & g_{k}(z(X, T))+\iint_{G} F_{k}\left(x, t, z(x, t), z_{x}(x, t), z_{t}(x, t), u(x, t)\right) d x d t \\
& +\int_{x_{0}}^{X} P_{k}(x, z(x, T)) d x+\int_{t_{0}}^{T} Q_{k}(t, z(X, t)) d t .
\end{aligned}
$$

In this case we replace assumption $\left(A_{4}\right)$ by the following.

$\left(\mathrm{A}_{4}^{\prime}\right)$ The functions $g_{k}: \mathbb{R}^{n} \rightarrow \mathbb{R}$ are twice continuously differentiable. The functions $F_{k}\left(x, t, z_{1}, z_{2}, z_{3}, u\right): G \times \mathbb{R}^{3 n} \times U \rightarrow \mathbb{R}$ are measurable on $G$ for fixed $\left(z_{1}, z_{2}, z_{3}, u\right)$, continuous on $U$ for fixed $\left(x, t, z_{1}, z_{2}, z_{3}\right)$, and twice continuously differentiable on $\mathbb{R}^{3 n}$ for fixed $(x, t, u)$. The functions $P_{k}(x, z):\left[x_{0}, X\right] \times \mathbb{R}^{n} \rightarrow$ $\mathbb{R}$ are measurable on $\left[x_{0}, X\right]$ for fixed $z$ and twice continuously differentiable on $\mathbb{R}^{n}$ for fixed $x$. The functions $Q_{k}(t, z):\left[t_{0}, T\right] \times \mathbb{R}^{n} \rightarrow \mathbb{B}$ are measurable on $\left[t_{0}, T\right]$ for fixed $z$ and twice continuously differentiable on $\mathbb{R}^{n}$ for fixed $t$. Furthermore, there is a function $K^{\prime}(x, t, u)$ with $K^{\prime}(x, t, u(x, t)) \in L_{\infty}(G)$, such that for $i=1, \ldots, n ; j=1,2,3$,

$$
\left|F_{z_{j}}\left(x, t, z, z_{x}, z_{t}, u\right)\right| \leq K^{\prime}(x, t, u(x, t)) .
$$

In this case, the Hamiltonian function $H^{(k)}$ is modified to

$$
\begin{aligned}
& H^{(k)}(x, t, Z, u, \lambda, \mu, p, q):= \\
& \quad \lambda^{(k)} z_{x}+\mu^{(k)} z_{t}+\left(p^{(k)}+q^{(k)}\right) f(x, t, Z, u)+F_{k}\left(x, t, z, z_{x}, z_{t}, u\right),
\end{aligned}
$$


where, as before, $Z:=\left(z, \zeta, z_{x}, \zeta_{x}, z_{t}, \zeta_{t}\right)$.

By minor modifications of developments in Sections 3-5, we obtain the following result, the details of proof of which are omitted.

THEOREM 6.1. Let $\hat{u} \in \Omega$ be an optimal control for problem $(P)$ with $J_{k}$ defined as in (6.1). Suppose that assumptions $\left(A_{1}\right)-\left(A_{3}\right),\left(A_{4}^{\prime}\right)$, and $\left(A_{6}\right)-\left(A_{7}\right)$ are satisfied. Then there exist a nonzero vector $\chi=\left(\chi_{0}, \chi_{1}, \ldots, \chi_{r}\right) \in \mathbb{R}^{1+r}$ with

$$
\chi_{k} \geq 0 \quad \text { for } k \in I ; \quad \chi_{k}=0 \quad \text { for } k \in\{0,1, \ldots, r\} \backslash I,
$$

and a set of multipliers $(\hat{\lambda}, \hat{\mu}, \hat{p}, \hat{q}) \in L_{\infty}\left(G, \mathbb{R}^{4 n}\right)$ such that the maximum principle

$$
\hat{H}(x, t) \leq \hat{H}(x, t ; v), \quad \text { for all } v \in U \text {, a.e. }(x, t) \in G
$$

holds, where $H$ is defined as in (6.2) and $\lambda, \mu, p, q$ satisfy (5.8)-(5.11), along with the boundary conditions

$$
\begin{array}{ll}
\hat{p}(X, t)=\sum_{k=0}^{r} \chi_{k}\left[\frac{1}{2} \frac{\partial g_{k}}{\partial z}(\hat{z}(X, T))\right]+\frac{\partial Q_{k}}{\partial z}(\hat{z}(X, t)), & t \in\left[t_{0}, T\right] \\
\hat{q}(x, T)=\sum_{k=0}^{r} \chi_{k}\left[\frac{1}{2} \frac{\partial g_{k}}{\partial z}(\hat{z}(X, T))+\frac{\partial P_{k}}{\partial z}(\hat{z}(x, T))\right], & x \in\left[x_{0}, X\right] .
\end{array}
$$

\section{A special case}

Under further regularity assumptions we derive a simplified version of Theorem 5.2 in this section. We require the following assumptions.

(A $A_{8}$ For a given optimal pair $(\hat{u}, \hat{z})$ and the corresponding multipliers $\lambda, \mu, p$, and $q$, the (generalized) partial derivatives

$$
\frac{\partial}{\partial x} \sum_{j=1}^{n}\left(p_{j}+q_{j}\right)\left(\frac{\partial f_{j}}{\partial z_{i x}}+\dot{\gamma} \frac{\partial f_{j}}{\partial \zeta_{i x}}\right), \quad \frac{\partial}{\partial t} \sum_{j=1}^{n}\left(p_{j}+q_{j}\right)\left(\frac{\partial f_{j}}{\partial z_{i t}}+\dot{\gamma} \frac{\partial f_{j}}{\partial \zeta_{i t}}\right)
$$

exist for $i=1,2, \ldots, n$. 
Under $\left(A_{8}\right)$, the adjoint equations (5.9)-(5.10) can be differentiated as follows.

$$
\begin{array}{ll}
\hat{p}_{x t}=-\hat{\mu}_{t}-\left[\left(\hat{f}_{z_{t}}(x, t)+\dot{\gamma}(t) \hat{f}_{\zeta_{t}}(x, \gamma(t))\right)(\hat{p}+\hat{q})\right]_{t}, \\
\hat{p}_{x t}=-\hat{\mu}_{t}-\left[\hat{f}_{z_{t}}(x, t)(\hat{p}+\hat{q})\right]_{t}, \quad(x, t) \in\left[x_{0}, X\right] \times\left[t_{0}, \sigma(T)\right] \\
\hat{q}_{x t}=-\hat{\lambda}_{x}-\left[\left(\hat{f}_{z_{x}}(x, t)+\dot{\gamma}(t) \hat{f}_{\zeta_{x}}(x, \gamma(t))\right)(\hat{p}+\hat{q})\right]_{t}, \\
\hat{p}_{x t}=-\hat{\lambda}_{x}-\left[\hat{f}_{z_{x}}(x, t)(\hat{p}+\hat{q})\right]_{t}, \quad & (x, t) \in\left[x_{0}, X\right] \times\left[t_{0}, \sigma(T)\right]
\end{array}
$$

Setting $\theta:=p+q$, and $\mathscr{H}:=\theta \cdot f$, we derive from the above equations and (5.8) that

$$
\begin{aligned}
\theta_{x t}= & \hat{\mathscr{H}}_{z}(x, t)+\dot{\gamma} \hat{\mathscr{H}}_{\zeta}(x, \gamma(t))-\left(\hat{\mathscr{H}}_{z_{x}}(x, t)+\dot{\gamma}(t) \hat{\mathscr{H}}_{\zeta_{x}}(x, \gamma(t))\right)_{x} \\
& -\left(\hat{H}_{z_{t}}(x, t)+\dot{\gamma}(t) \hat{\mathscr{H}}_{\zeta_{t}}(x, \gamma(t))\right)_{t}, \quad(x, t) \in\left[x_{0}, X\right] \times\left[t_{0}, \sigma(T)\right] \\
\theta_{x t}= & \hat{\mathscr{H}}_{z}(x, t)-\left(\hat{\mathscr{H}}_{z_{x}}(x, t)\right)_{x}-\left(\hat{\mathscr{H}}_{z_{t}}(x, t)\right)_{t}, \quad(x, t) \in\left[x_{0}, X\right] \times[\sigma(T), T] .
\end{aligned}
$$

Regarding the boundary conditions for $\theta$, we have from (5.11)-(5.13) that

$$
\begin{aligned}
\theta_{x}(x, T) & =\hat{p}_{x}(x, T)+\hat{q}_{x}(x, T)=\hat{p}_{x}(x, T) \\
& =-\hat{\mu}(x, T)-\hat{f}_{z_{t}}(x, T) \theta(x, T)=-\hat{f}_{z_{t}}(x, T) \theta(x, T), \quad x \in\left[x_{0}, X\right] \\
& =-\hat{\mathscr{H}}_{z_{t}}(x, T) .
\end{aligned}
$$

Similarly

$$
\begin{aligned}
\theta_{t}(X, t) & =-\left(\hat{f}_{z_{x}}(X, t)+\dot{\gamma}(t) \hat{f}_{\zeta_{x}}(X, \gamma(t))\right)(\hat{p}+\hat{q}) \\
& =-\hat{H}_{z_{x}}(X, t)-\dot{\gamma}(t) \hat{H}_{\zeta_{x}}(X, \gamma(t)), \quad t \in\left[t_{0}, \sigma(T)\right],
\end{aligned}
$$

and

$$
\theta_{t}(X, t)=-\hat{f}_{z_{x}}(X, t) \theta(X, t)=-\hat{\mathscr{H}}_{z_{x}}(X, t) \quad t \in[\sigma(T), T]
$$

Finally,

$$
\theta(X, T)=\sum_{k=0}^{r} \chi_{k} \frac{\partial g_{k}}{\partial z}(\hat{z}(X, T)) .
$$

Summarizing the above developments, we have the following results. 
THEOREM 7.1. Let $\hat{u} \in \Omega$ be an optimal control for problem $(P)$. Suppose that assumptions $\left(A_{1}\right)-\left(A_{8}\right)$ are satisfied. Then there exist a nonzero vector $\chi=\left(\chi_{0}, \chi_{1}, \ldots, \chi_{r}\right) \in$ $\mathbb{R}^{1+r}$ with

$$
\chi_{k} \geq 0 \quad \text { for } k \in I ; \quad \chi_{k}=0 \text { for } f \in\{0,1, \ldots, r\} \backslash I
$$

and a unique $\hat{\theta} \in L_{\infty}\left(G, \mathbb{R}^{n}\right)$ such that

$$
\begin{gathered}
\hat{\mathscr{H}}(x, t, \hat{Z}(x, t), \hat{u}(x, t), \hat{\theta}(x, t)) \leq \mathscr{H}(x, t, \hat{Z}(x, t), v, \hat{\theta}(x, t)) \\
\text { for all } v \in U \text { and a.e. }(x, t) \in G,
\end{gathered}
$$

where

$$
\begin{array}{rlrl} 
& \mathscr{H}(x, t, Z, u, \theta):=\theta \cdot f(x, t, Z, u), \\
\hat{\theta}_{x t}= & \hat{\mathscr{H}}_{z}(x, t)+\dot{\gamma}(t) \hat{\mathscr{H}}_{\zeta}(x, \gamma(t))-\left(\hat{\mathscr{H}}_{z_{x}}(x, t)+\dot{\gamma}(t) \hat{\mathscr{H}}_{\zeta_{x}}(x, \gamma(t))\right)_{x} \\
& -\left(\hat{\mathscr{H}}_{z_{t}}(x, t)+\dot{\gamma}(t) \hat{\mathscr{H}}_{\zeta_{t}}(x, \gamma(t))\right)_{t}, & (x, t) \in\left[x_{0}, X\right] \times\left[t_{0}, \sigma(T)\right] \\
\hat{\theta}_{x t}= & \hat{\mathscr{H}}_{z}(x, t)-\left(\hat{\mathscr{H}}_{z_{x}}(x, t)\right)_{x}-\left(\hat{\mathscr{H}}_{z_{t}}(x, t)\right)_{t}, & (x, t) \in\left[x_{0}, X\right] \times[\sigma(T), T] \\
\hat{\theta}_{x}(x, T)=-\hat{\mathscr{H}}_{z_{t}}(x, T), & x \in\left[x_{0}, X\right] \\
\hat{\theta}_{t}(X, t)=-\hat{\mathscr{H}}_{z_{x}}(X, t)-\dot{\gamma}(t) \hat{\mathscr{H}}_{\zeta_{x}}(X, \gamma(t)), & t \in\left[t_{0}, \sigma(T)\right] \\
\hat{\theta}_{t}(X, t)=-\hat{\mathscr{H}}_{z_{x}}(X, t), & t \in[\sigma(T), T] \\
\hat{\theta}(X, T)= & \sum_{k=0}^{r} \chi_{k} \frac{\partial g_{k}}{\partial z}(\hat{z}(X, T)) .
\end{array}
$$

REMARK 7.1. A very special case of problem (P) is considered in [9] and a result similar to Theorem 7.1 is established. Specifically, in [9], $f$ is a scalar function, controls are assumed piecewise continuous, there are no terminal state constraints, $g(z)=z$, and a simple delay problem is considered.

\section{Conclusion}

In this paper we have presented an approach to (first order) necessary conditions, in the form of a Pontryagin maximum principle, for constrained controlled processes with distributed parameters and deviating argument. The approach presented here applies equally well to other nonlinear constrained distributed parameter systems with deviating argument.

\section{Acknowledgement}

The author is thankful to the referees for their helpful comments.

This work was supported, in part, by funds provided by the University of North Carolina at Charlotte. 


\section{References}

[1] N. U. Ahmed and K. L. Teo, Optimal control of distributed parameter systems (Elsevier North Holland, New York, 1981).

[2] E. Casas, "Boundary control of semilinear elliptic equations with pointwise state constraints", SIAM J. Control Optim. 31 (1993) 993-1006.

[3] M. Giorecki et al., Analysis and synthesis of time delay systems (John Wiley \& Sons, New York, 1991).

[4] M. A. Kazemi, "Optimal control of systems governed by partial differential equations with integral inequality constraints", Nonlinear Analysis, Theory, Methods, Appl. 8 (1984) 1409-1425.

[5] M. A. Kazemi, "Optimal control of distributed hyperbolic systems with time delay", Far East $J$. Math. Sci. I (1993) 53-68.

[6] A. Kowalewski, "Optimal control of distributed parabolic systems involving time lags", IMA J. Math Control. Infor. 7 (1991) 375-393.

[7] J. L. Lions, Optimal control of systems governed by partial differential equations (Springer-Verlag, Berlin, 1971).

[8] V. I. Plotnikov and V. I. Sumin, "Optimization of objects with distributed parameters described by Goursah-Darboux systems", Comput. Maths. Math. Phys. 12 (1972) 67-77.

[9] L. S. Sadek, "Optimal control of time-delay systems with distributed parameters", J. Optim. Theory Appl. 67 (1990) 567-585.

[10] M. E. Salukvadze and M. T. Teuteunava, "An optimal control problem for systems described by partial differential equations of hyperbolic type with delay", J. Optim. Theory Appl. 52 (1987) 311-322.

[11] M. B. Suryanarayana, "Necessary conditions for optimization problems with hyperbolic partial differential equations", SIAM J. Control II (1973) 130-147.

[12] K. L. Teo, "Optimal control of systems governed by time delayed second order linear parabolic partial differential equations with a first boundary condition", J. Optim. Theory Appl. 29 (1979) $437-481$.

[13] O. V. Vasil'ev and V. A. Srochko, "Optimization of a class of controlled processes with distributed parameters", Sibirian Math.J. 19 (1978) 328-331.

[14] P. K. C. Wang, "Optimal control of parabolic systems with boundary conditions having time delays”, SIAM J. Control optim. 13 (1975) 274-293.

[15] R. L. Wheeden and A. Zygmund, Measure and integral (Marcel Dekker, New York, 1977).

[16] K. H. Wong, "Optimal control computation for parabolic systems with boundary conditions involving time delays", J. Optim. Theory Appl. 53 (1987) 475-507.

[17] Z. S. Wu and K. L. Teo, Computational methods for optimizing distributed systems (Academic Press, 1984).

[18] Z. S. Wu and K. L. Teo, "A conditional gradient method for an optimal control problem involving a class of nonlinear second order hyperbolic partial differential equations", J. Math. Anal. Appl. 91 (1983) 376-393.

[19] Z. S. Wu and K. L. Teo, "A convex optimal control problem involving a class of linear hyperbolic systems", J. Optim. Theory App. 39 (1983) 341-560. 